\title{
The complete mitochondrial genome of the scab mite Psoroptes cuniculi (Arthropoda: Arachnida) provides insights into Acari phylogeny
}

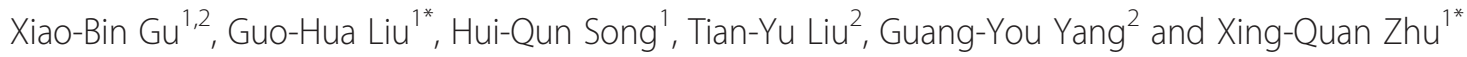

\begin{abstract}
Background: Limited available sequence information has greatly impeded population genetics, phylogenetics and systematics studies in the subclass Acari (mites and ticks). Mitochondrial (mt) DNA is well known to provide genetic markers for investigations in these areas, but complete mt genomic data have been lacking for many Acari species. Herein, we present the complete mt genome of the scab mite Psoroptes cuniculi.

Methods: P. cuniculi was collected from a naturally infected New Zealand white rabbit from China and identified by morphological criteria. The complete mt genome of $P$. cuniculi was amplified by PCR and then sequenced. The relationships of this scab mite with selected members of the Acari were assessed by phylogenetic analysis of concatenated amino acid sequence datasets by Bayesian inference (BI), maximum likelihood (ML) and maximum parsimony (MP).

Results: This mt genome (14,247 bp) is circular and consists of 37 genes, including 13 genes for proteins, 22 genes for tRNA, 2 genes for rRNA. The gene arrangement in $m t$ genome of $P$. cuniculi is the same as those of Dermatophagoides farinae (Pyroglyphidae) and Aleuroglyphus ovatus (Acaridae), but distinct from those of Steganacarus magnus

(Steganacaridae) and Panonychus citri (Tetranychidae). Phylogenetic analyses using concatenated amino acid sequences of 12 protein-coding genes, with three different computational algorithms (BI, ML and MP), showed the division of subclass Acari into two superorders, supported the monophylies of the both superorders Parasitiformes and Acariformes; and the three orders Ixodida and Mesostigmata and Astigmata, but rejected the monophyly of the order Prostigmata.

Conclusions: The mt genome of $P$. cuniculi represents the first $\mathrm{mt}$ genome of any member of the family Psoroptidae. Analysis of $\mathrm{mt}$ genome sequences in the present study has provided new insights into the phylogenetic relationships among several major lineages of Acari species.
\end{abstract}

Keywords: Psoroptes cuniculi, Mitochondrial genome, Mitochondrial DNA, Phylogenetic analyses

\section{Background}

The metazoan mitochondrial $(\mathrm{mt})$ genome possess a circular double strand DNA that varies in size from 15 to $20 \mathrm{~kb}$, generally encoding for 36-37 genes: $12-13$ protein-coding genes (PCGs), two ribosomal RNAs (rRNA) genes and 22 transfer RNAs (tRNA) [1,2]. Mt genomes have been extensively used as genetic markers in molecular phylogenetic studies due to it having several useful properties

\footnotetext{
* Correspondence: liuguohua5202008@163.com; xingquanzhu1@hotmail.com 'State Key Laboratory of Veterinary Etiological Biology, Key Laboratory of Veterinary Parasitology of Gansu Province, Lanzhou Veterinary Research Institute, Chinese Academy of Agricultural Sciences, Lanzhou, Gansu Province 730046, P R China

Full list of author information is available at the end of the article
}

(i.e., haploidy, compactness, maternal inheritance, relatively high mutation rates, and the lack of recombination) [3]. To date, there are over 4000 complete $\mathrm{mt}$ genome sequences of metazoans available in GenBank, including some from parasites [4-10]. In spite of the availability of advanced DNA technologies, however, there is still major gap in our knowledge of $\mathrm{mt}$ genomes of the subclass Acari.

Currently, the Acari encompasses over 55,000 species (ticks and mites) and forms the largest group among the class Arachnida [11]. Many species of ticks and mites threaten the health of plants, animals and humans on a global scale, causing major economic losses and significant 
public health problems [12,13]. Despite their abundance, medical, veterinary and economic significance, the genetics, epidemiology, biology and phylogenetic relationships in this group remain poorly understood. Acari were traditionally classified into three monophyletic lineages: superorders Parasitiformes (=Anactinotrichida), Opiloacariformes and Acariformes (=Actinotrichida) [14]. However, phylogenetic relationships among Acari have been revised in the past using nuclear rDNA gene sequences, indicating Opiloacariformes + Parasitiformes as the second monophyletic lineage of Acari [15,16]. Recently, mt genome sequences have been used to understand the phylogenetic relationships among Acari. Dermauw et al. [17] inferred the Acari phylogeny with the mt genome sequences of 21 species. Liu et al. [8] and Burger et al. [4] inferred the phylogeny of ticks with the mt genome sequences of 16 and 21 species, respectively. A difference among mt genome phylogenies is on the relationship within the order Ixodida. Dermauw et al. [17] and Burger et al. [4] showed strong support that Metastriate + Prostriate are sister to Ornithodorinae. However, Liu et al. [8] showed strong support that Ornithodorinae is more closely related to Prostriate, and Metastriate is sister to Ornithodorinae + Prostriate. More recently, Burger et al. [18] showed that Metastriate + Prostriate are sister to Ornithodorinae based on nuclear rDNA and mt gene sequences. The systematics and classification of the Acari are controversial in these analyses due to limited or underrepresented taxon sampling. So, the phylogenetic relationships inferred from nuclear and mt sequences need to be retested with more taxa from a wide range of lineages.

In the present study, we sequenced the complete mt genome of the scab mite $P$. cuniculi in the family Psoroptidae of the order Astigmata. P. cuniculi is a common worldwide parasite of rabbits, causing considerable economic loss due to weight loss, decreased food consumption, vestibular dysfunction, meningitis and death [19]. Also, we inferred the phylogenetic relationships with the concatenated mt amino acid sequences of $P$. cuniculi and 50 other Acari species that have been sequenced to date.

\section{Methods}

\section{Ethics statement}

This study was approved by the Animal Ethics Committee of The Lanzhou Veterinary Research Institute, Chinese Academy of Agricultural Sciences (Permit code. LVRIAEC2013006). The New Zealand white rabbit, from which $P$. cuniculi was collected, was handled in accordance with good animal practices required by the Animal Ethics Procedures and Guidelines of the People's Republic of China.

\section{Parasites and DNA extraction}

$P$. cuniculi samples were collected from a naturally infected New Zealand white rabbit in Sichuan Province, China. The adult mites were isolated from ear cerumens of the rabbit, according to the previous descriptions [20]. The presence of mites was detected by light microscopic examination, and identification of $P$. cuniculi was conducted by morphological criteria and site of predilection [21], then fixed in $70 \%$ alcohol and stored at $-20^{\circ} \mathrm{C}$ until use. Total genomic DNA was isolated from these specimens using sodium dodecyl sulphate/proteinase $\mathrm{K}$ treatment, followed by spincolumn purification (Wizard ${ }^{\mathrm{SV}}$ Genomic DNA Purification System, Promega). In brief, P. cuniculi collected from the same host were ground in a mortar and subsequently suspended in $275 \mu \mathrm{L}$ lysis buffer $(200 \mu \mathrm{L}$ Nuclei Lysis Solution, $50 \mu \mathrm{L}$ EDTA, $20 \mu \mathrm{L}$ proteinase $\mathrm{K}$ and $5 \mu \mathrm{L}$ RNase A Solution). After vortexing, the sample was incubated at $55^{\circ} \mathrm{C}$ for 16-18 h, and the DNA was extracted by shaking with $250 \mu \mathrm{L}$ Wizard SV Lysis Buffer. After centrifugation, the DNA was washed, eluted and stored.

\section{PCR amplification and sequencing}

Nine pairs of PCR primers were used to amplify overlapping segments of the complete mt genome of $P$. cuniculi as shown in Table 1. Briefly, the segment of $\operatorname{cox} 1$ was amplified using the primers COIF/COIR [22]. The other segments were amplified using designed primers (Table 1) based on sequences well conserved in the Dermatophagoides farinae and D. pteronyssinus [17,23]. All PCR reactions $(25 \mu \mathrm{L})$ were performed in $2.5 \mathrm{mM}$ dNTP mixture, $2.5 \mu \mathrm{L} 10 \times$ Ex Taq buffer $\left(\mathrm{Mg}^{2+}\right.$ Plus), $10 \mathrm{pmol}$ of each primer, $1 \mathrm{U}$ Ex Taq polymerase (Takara), $1 \mu \mathrm{L}$ of DNA

\begin{tabular}{|c|c|}
\hline Primer & Sequence $\left(5^{\prime}\right.$ to $\left.3^{\prime}\right)$ \\
\hline COIF & GGTCAACAAATCATAAAGATATTGG \\
\hline COIR & TAAACTTCAGGGTGACCAAAAAATC \\
\hline COI-COIIF & TТAATTCTACATTCTTTGACC \\
\hline COI-COIIR & GGAGAACTAGAATCTTGAAA \\
\hline COII-ND3R & TITCAAGATTCTAGTTCTCC \\
\hline COII-ND3F & ACTCATAACARGTGGCTAAT \\
\hline ND3-12SR & TTCTATTAGCCACCTGTT \\
\hline ND3-12SF & AGTAACTCATTGGAAACC \\
\hline 12S-ND1R & AAACTAGGATTAGATACCA \\
\hline 12S-ND1F & ATTGCTTITITTACWTTAGT \\
\hline ND1-ND4F & GCAGATAGGCTTATGACG \\
\hline ND1-ND4R & AGAAAACAAAATAAAGGTAGG \\
\hline ND4F & TGRCTTCCTAAAGCTCATG \\
\hline ND4R & ATCTCAGAAAAAAAVGWTATAAAA \\
\hline ND4-CytbF & TAGTGTTCATCTTTGACTTCC \\
\hline ND4-CytbR & TACGCCATITTACGGGCAATTCC \\
\hline Cytb-COIF & CAGGGTTTGCTCCAATTCATGT \\
\hline Cytb-COIR & CCCCTAAAATTGAAGAAATACCAGC \\
\hline
\end{tabular}




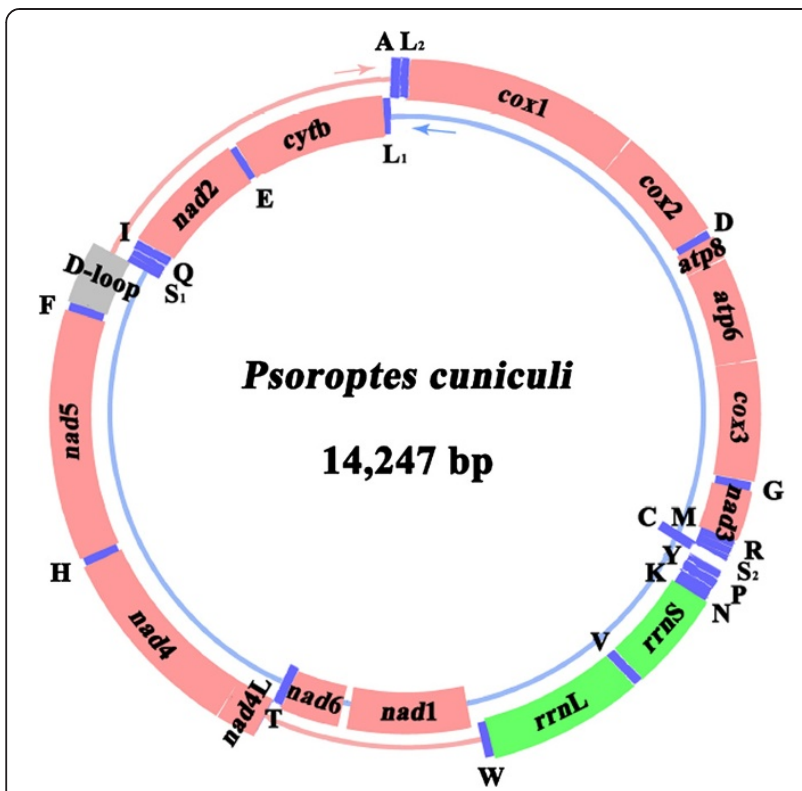

Figure 1 Arrangement of the mitochondrial genome of Psoroptes cuniculi. Gene scaling is only approximate. All genes have standard nomenclature including the 22 tRNA genes, which are designated by the one-letter code for the corresponding amino acid, with numerals differentiating each of the two leucine- and serine-specifying tRNAs ( $L_{1}$ and $L_{2}$ for codon families CUN and UUR, respectively; $S_{1}$ and $S_{2}$ for codon families $A G N$ and $U C N$, respectively).

sample in a thermocycler (Eppendorf, Germany) under the following conditions: $94^{\circ} \mathrm{C}$ for $5 \mathrm{~min}$ (initial denaturation); then followed by 35 cycles of $94^{\circ} \mathrm{C}$ for $30 \mathrm{~s}$ (denaturation), $36 \sim 60^{\circ} \mathrm{C}$ for $30 \mathrm{~s}$ (annealing), and $68^{\circ} \mathrm{C}$ for $1 \sim 4.5 \mathrm{~min}$ (extension) according to the product length; with a final extension step at $68^{\circ} \mathrm{C}$ for $10 \mathrm{~min}$. Each PCR reaction yielded a single band as detected in a $1 \%(\mathrm{~W} / \mathrm{V})$ agarose gel upon ethidium-bromide staining (not shown). PCR products were subsequently sent to Invitrogen Biotechnology Company (Shanghai, China) for sequencing using a primerwalking strategy.

\section{Sequence analyses}

Sequences were assembled manually and aligned against the complete $\mathrm{mt}$ genome sequences of $D$. farinae available using the computer program MAFFT 7.122 [24] to identify gene boundaries. Each gene was translated into amino acid sequence using the invertebrate mitochondrial genetic code in MEGA 5 [25], and aligned based on its amino acid sequence using default settings. The translation initiation and termination codons were identified to avoid gene overlap and to optimize the similarity between the gene lengths of closely-related mite $\mathrm{mt}$ genomes. For analyzing tRNA genes, the program tRNAscan-SE [26] and the program ARWEN [27] were used to detect tRNA and infer their secondary structure. For tRNAscan-SE, the following parameters were changed: Source: $=$ Nematode Mito, Search Mode $=$ tRNAscan $>$ Cove, Genetic Code $=$ Invertebrate Mito, Cove score cutoff $=0.1$. EufindtRNA search parameters $=$ relaxed. Putative secondary structures of 17 tRNA genes were identified, the remaining five tRNA genes (tRNA-Ser ${ }^{\text {UCN }}$, tRNA-Ile, tRNA-Pro, tRNA$\mathrm{Val}$ and tRNA-Ala) were identified by recognizing potential secondary structures and anticodon sequences by eye, and two rRNA genes were predicted by comparison with those of other mites previously reported $[17,23]$.

Table 2 Composition of mitochondrial genomes in the superorder Acariformes

\begin{tabular}{|c|c|c|c|c|c|c|c|}
\hline Species & Size (bp) & Accession number & $\mathrm{A} \%$ & $\mathrm{~T} \%$ & G\% & $\mathrm{C} \%$ & $\mathrm{~A}+\mathrm{T} \%$ \\
\hline Aleuroglyphus ovatus & 14328 & NC_023778 & 23.6 & 44.7 & 18.7 & 13.0 & 68.3 \\
\hline Ascoschoengastia sp. & 16067 & NC_010596 & 35.6 & 34.5 & 14.2 & 15.7 & 70.1 \\
\hline Dermatophagoides farinae & 14266 & NC_013184 & 26.7 & 44.7 & 17.6 & 11.0 & 71.4 \\
\hline Dermatophagoides pteronyssinus & 14203 & NC_012218 & 29.1 & 43.5 & 16.4 & 11.0 & 72.6 \\
\hline Leptotrombidium akamushi & 13698 & NC_007601 & 33.2 & 34.3 & 15.0 & 17.5 & 67.5 \\
\hline Leptotrombidium deliense & 13731 & NC_007600 & 34.4 & 35.6 & 14.1 & 15.9 & 70.0 \\
\hline Leptotrombidium pallidum & 16779 & NC_007177 & 34.4 & 36.6 & 13.9 & 15.1 & 71.0 \\
\hline Panonychus citri & 13075 & NC_014347 & 39.5 & 46.0 & 7.5 & 7.0 & 85.5 \\
\hline Panonychus ulmi & 13115 & NC_012571 & 40.3 & 45.3 & 7.2 & 7.2 & 85.6 \\
\hline Psoroptes cuniculi & 14247 & KJ957822 & 29.4 & 41.7 & 16.8 & 12.1 & 71.1 \\
\hline Steganacarus magnus & 13818 & NC_011574 & 36.5 & 38.1 & 12.2 & 13.2 & 74.6 \\
\hline Tetranychus cinnabarinus & 13092 & NC_014399 & 43.5 & 40.9 & 7.6 & 8.0 & 84.4 \\
\hline Tetranychus urticae & 13103 & NC_010526 & 43.2 & 41.1 & 7.7 & 8.0 & 84.3 \\
\hline Unionicola foili & 14738 & NC_011036 & 43.8 & 29.2 & 9.8 & 17.3 & 73.0 \\
\hline Unionicola parkeri & 14734 & NC_014683 & 46.6 & 26.2 & 9.9 & 17.3 & 72.8 \\
\hline Walchia hayashii & 14857 & NC_010595 & 46.1 & 26.9 & 9.4 & 17.6 & 73.0 \\
\hline
\end{tabular}




\section{Phylogenetic analyses}

For comparative purposes, amino acid sequences predicted from published $\mathrm{mt}$ genomes of 50 Acari species were also included in the present analysis, using the scorpion Centruroides limpidus (GenBank accession number NC_006896) as an outgroup [28]. The 12 amino acid sequences (not ATP8) were single aligned using MAFFT 7.122 and then concatenated, and ambiguously aligned regions were excluded using Gblocks 0.91b (doc) with the default parameters [29] using the options for a less stringent selection. Phylogenetic analyses were conducted using three methods: Bayesian inference (BI), Maximum

Table 3 Organization of Psoroptes cuniculi mitochondrial genome

\begin{tabular}{|c|c|c|c|c|c|}
\hline Genes/regions & Position & Size (bp) & Strand & Start codons & Stop codons \\
\hline $\operatorname{cox} 1$ & $1-1548$ & 1548 & J & ATA & TAA \\
\hline $\cos 2$ & $1562-2330$ & 769 & J & ATG & $\mathrm{T}$ \\
\hline tRNA- Asp (D) & $2332-2385$ & 54 & J & & \\
\hline atp8 & $2397-2543$ & 147 & J & ATA & TAG \\
\hline atp6 & $2550-3221$ & 672 & J & ATG & TAA \\
\hline $\operatorname{cox} 3$ & $3228-4010$ & 783 & J & ATG & TAA \\
\hline tRNA-Gly (G) & $4014-4069$ & 56 & J & & \\
\hline nad3 & 4075-4393 & 319 & J & ATA & $\mathrm{T}$ \\
\hline tRNA-Arg (R) & 4395-4449 & 55 & J & & \\
\hline tRNA-Met (M) & $4454-4505$ & 52 & J & & \\
\hline tRNA-Ser $^{\mathrm{UCN}}(\mathrm{S} 2)$ & $4506-4557$ & 52 & J & & \\
\hline tRNA-Cys (C) & $4560-4609$ & 50 & $N$ & & \\
\hline tRNA-Pro (P) & $4619-4674$ & 56 & J & & \\
\hline tRNA-Tyr $(Y)$ & $4675-4728$ & 54 & J & & \\
\hline tRNA-Lys (K) & $4719-4780$ & 62 & J & & \\
\hline tRNA-Asn (N) & $4780-4837$ & 58 & J & & \\
\hline$r r n S$ & 4838-5495 & 658 & J & & \\
\hline tRNA-Val (V) & $5496-5547$ & 52 & J & & \\
\hline$r r n \mathrm{~L}$ & $5548-6571$ & 1024 & J & & \\
\hline tRNA-Trp (W) & $6572-6628$ & 57 & J & & \\
\hline nad1 & $6690-7613$ & 924 & $N$ & ATT & TAA \\
\hline nad6 & 7625-8071 & 447 & $\mathrm{~N}$ & ATA & TAG \\
\hline tRNA-Thr (T) & $8074-8129$ & 56 & $N$ & & \\
\hline nad4L & 8136-8405 & 270 & J & ATG & TAA \\
\hline nad4 & 8406-9699 & 1294 & J & ATG & $\mathrm{T}$ \\
\hline tRNA-His $(\mathrm{H})$ & $9700-9754$ & 55 & J & & \\
\hline nad5 & 9775-11392 & 1618 & J & ATT & $\mathrm{T}$ \\
\hline tRNA-Phe (F) & 11393-11446 & 54 & J & & \\
\hline D-loop & 11447-11828 & 382 & J & & \\
\hline tRNA-Ser ${ }^{A G N}(S 1)$ & 11829-11881 & 53 & N & & \\
\hline tRNA-GIn (Q) & 11880-11931 & 52 & $\mathrm{~N}$ & & \\
\hline tRNA- Ile (I) & 11935-11987 & 53 & $N$ & & \\
\hline nad2 & $12012-12912$ & 901 & $N$ & $T T G$ & $\mathrm{~T}$ \\
\hline tRNA-Glu (E) & 12918-12970 & 53 & $\mathrm{~N}$ & & \\
\hline cytb & 12971-14070 & 1100 & $\mathrm{~N}$ & ATG & TA \\
\hline tRNA-Leu ${ }^{\mathrm{CUN}}(\mathrm{L} 1)$ & 14080-14135 & 56 & N & & \\
\hline tRNA-Ala (A) & 14140-14189 & 50 & J & & \\
\hline tRNA-Leu $^{\text {UUR }}\left(L_{2}\right)$ & 14189-14245 & 57 & J & & \\
\hline
\end{tabular}


likelihood (ML) and Maximum parsimony (MP). The MtArt + I + G + F model of amino acid evolution was selected as the most suitable model of evolution by ProtTest 2.4 [30] based on the Akaike information criterion (AIC). As MtArt model is not implemented in the current version of MrBayes, an alternative model, MtREV, was used in BI and four chains (three heated and one cold) were run simultaneously for the Monte Carlo Markov Chain. Two independent runs for 1,000,000 metropolis-coupled MCMC generations, sampling a tree every 100 generation in MrBayes 3.1.1 [31]; the first 2,500 trees represented burn-in and the remaining trees were used to calculate Bayesian posterior probabilities (Bpp). The analysis was performed until the potential scale reduction factor approached 1 and the average standard deviation of split frequencies was less than 0.01. ML analysis was performed with PhyML 3.0 [32] using the subtree pruning and regrafting (SPR) method with a BioNJ starting tree, and the MtArt model of amino acid substitution with proportion of invariant sites (I) and gamma distribution (G) parameters estimated from the data with four discretized substitution rate classes, the middle of which was estimated using the median. Bootstrap frequency (Bf) was calculated using 100 bootstrap replicates. MP analysis was conducted using PAUP 4.0 Beta 10 program [33], with indels treated as missing character states; 1,000 random additional searches were performed using TBR. Bf was calculated using 1,000 bootstrap replicates, and 100 random

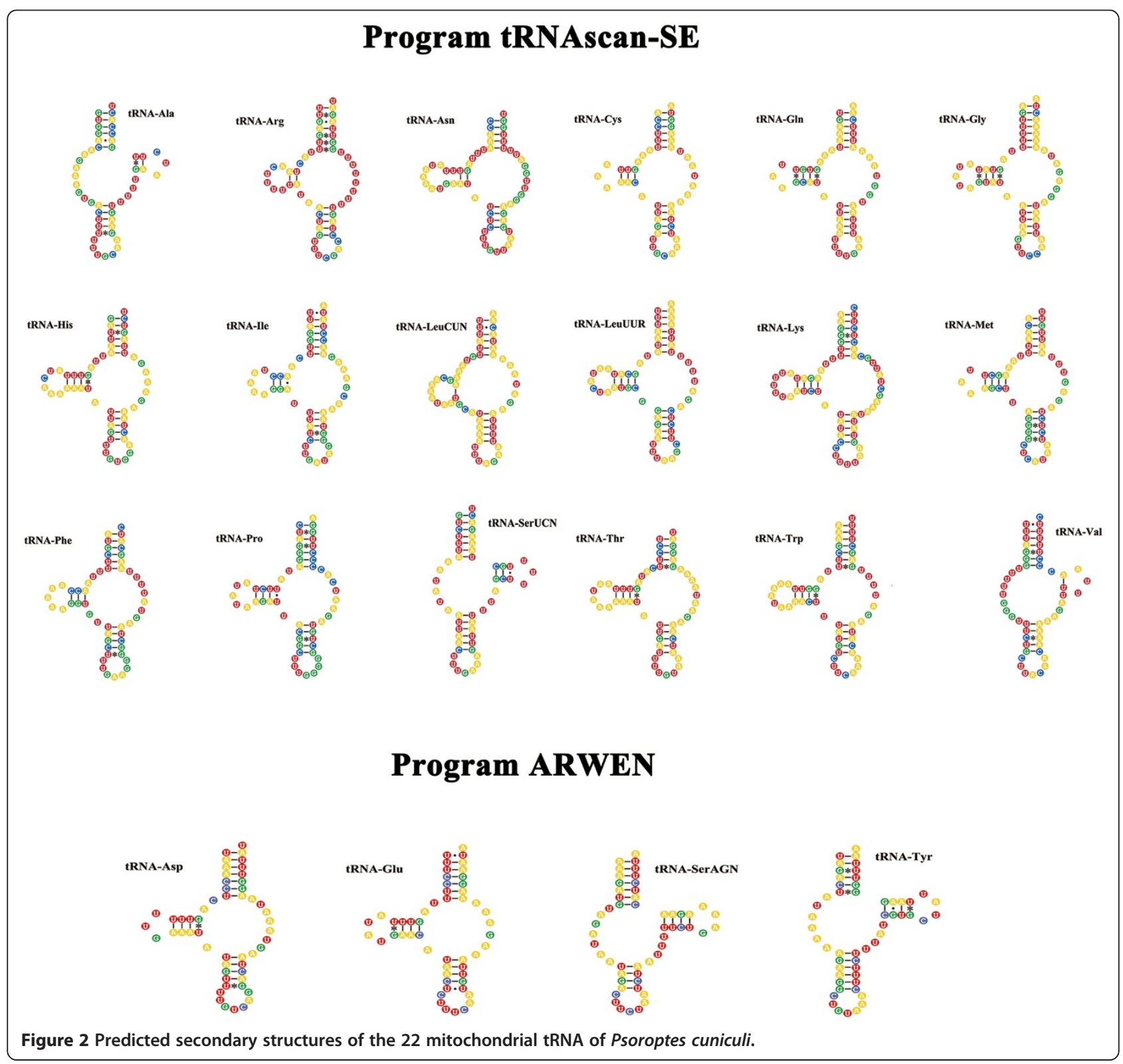


taxon additions in PAUP. Phylograms were drawn using the program FigTree v.1.4.

\section{Results}

\section{General features of the $\mathrm{mt}$ genome of $P$. cuniculi}

The complete mt genome of $P$. cuniculi was $14,247 \mathrm{bp}$ in length (Figure 1). This size is within the range of other mite $\mathrm{mt}$ genomes (Table 2). The sequences have been deposited in GenBank under the accession number KJ957822. This circular mt genome contains 13 PCGs (cox1-3, nad1-6, nad4L, cytb, atp6 and atp8), 22 tRNA genes, two rRNA genes and one D-loop region (Table 3). The genes are transcribed in both different directions. Except for four PCGs (nad1, nad6, nad2 and cytb) and seven tRNA genes (tRNA-Cys, tRNA-Thr, tRNA-Ser ${ }^{\mathrm{AGN}}$, tRNAGln, tRNA- Ile, tRNA-Glu and tRNA-Leu ${ }^{\mathrm{CUN}}$ ) encoded on the minority strand ( $\mathrm{N}$-strand), all other genes were encoded on the majority strand (J-strand) (Figure 1). The gene arrangement is the same as those of $D$. farinae (Pyroglyphidae) and Aleuroglyphus ovatus (Acaridae), but distinct from those of Steganacarus magnus (Steganacaridae) and Panonychus citri (Tetranychidae). The nucleotide composition of the complete mt genome of $P$. cuniculi is $\mathrm{A}=29.4 \%, \mathrm{~T}=41.7 \%, \mathrm{G}=16.8 \%$ and $\mathrm{C}=12.1 \%$, with a typically high $\mathrm{A}+\mathrm{T}$ content of $71.1 \%$ within the range of values found in $D$. farinae (71.4\%) and $D$. pteronyssinus (72.6\%) (Table 2).

\section{Annotation}

The length of PCGs of $P$. cuniculi was in the following order: nad5 $>\operatorname{cox} 1>\operatorname{nad} 4>\operatorname{cytb}>\operatorname{nad} 1>\operatorname{nad} 2>\operatorname{cox} 3>$ $\operatorname{cox} 2>$ atp $6>$ nad $6>$ nad $3>$ nad $4 \mathrm{~L}>\operatorname{atp} 8$ (Table 3). A total of 3588 amino acids are encoded in the $\mathrm{mt}$ genome of P. cuniculi. In this mt genome, 4 genes (cox1, nad 3 , atp 8 and nad6) use ATA, 6 genes (cox2, cox3, atp6, nad4, nad4L and $c y t \mathrm{~b})$ use ATG, 2 genes (nad1, nad5) use ATT and 1 gene (nad2) uses TTG as start codon, respectively (Table 3 ). All genes have complete termination codon except for $c y t b$, $\operatorname{cox} 2$, nad3, nad5, nad2 and nad4 genes which use abbreviated stop codon T or TA, 5 genes (cox1, $\operatorname{cox} 3$, nad1, nad4L and atp6) use TAA and 2 genes ( $a t p 8$ and nad6) use TAG as termination codon, respectively (Table 3). The $r r n S$ of $P$. cuniculi is located between tRNA-Asn and tRNA-Val, and $r r n \mathrm{~L}$ is located between tRNA-Val and tRNA-Trp. The length of the $r r n S$ gene is $658 \mathrm{bp}$ and $r r n \mathrm{~L}$ gene is $1024 \mathrm{bp}$ (Table 3). The $\mathrm{A}+\mathrm{T}$ contents of the $r r n \mathrm{~S}$ and $r r n \mathrm{~L}$ are $72.4 \%$ and $72.2 \%$, respectively. As the mt genomes of Acariform mites can contain short minimal tRNA genes [23],

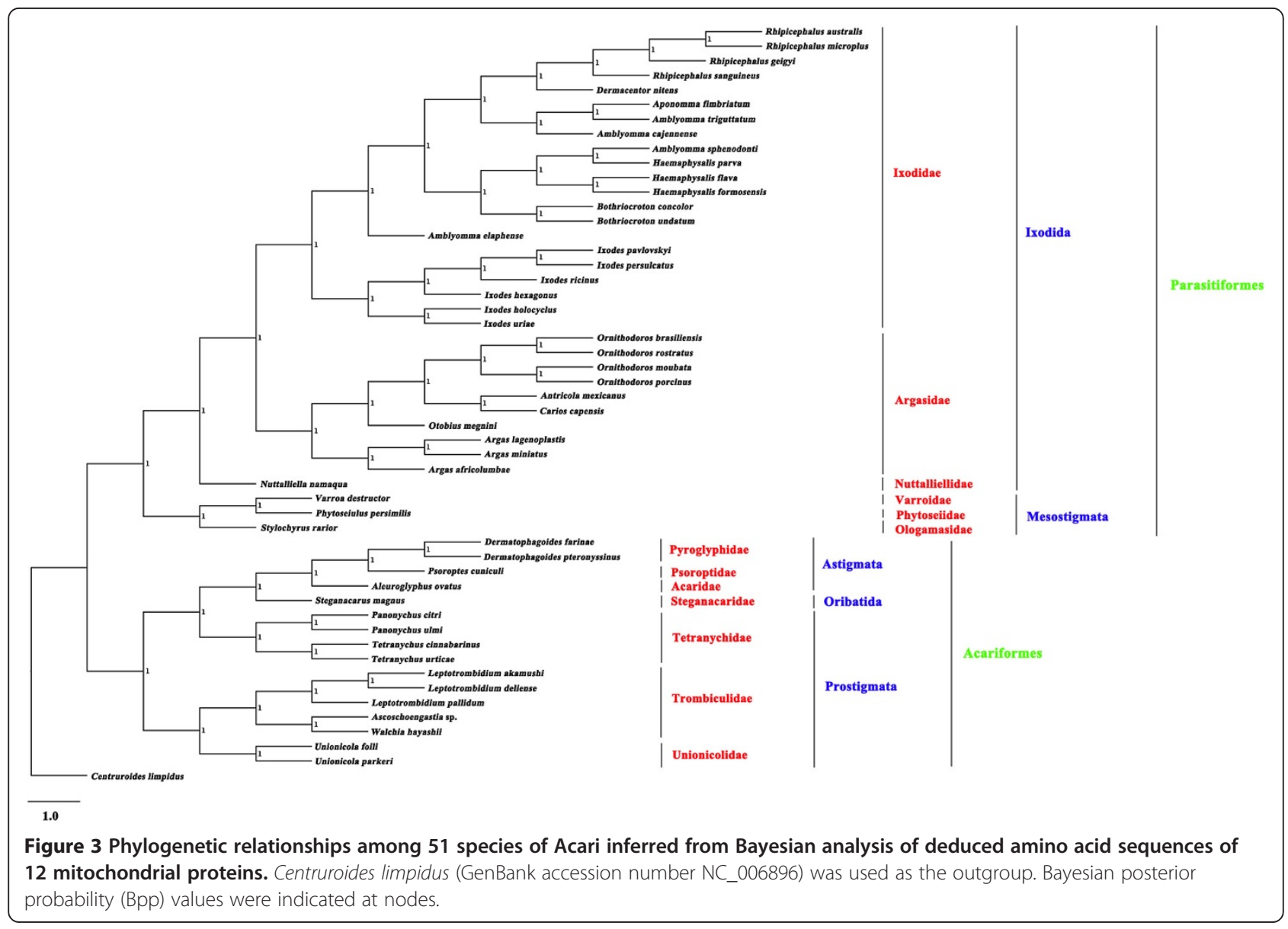


the identification of tRNA genes can sometimes be challenging. A total of 22 tRNA sequences were identified in the $P$. cuniculi mt genome, ranging from 50-62 bp (Table 3). Almost all tRNA genes are atypical lacking either the T- or Dloop. Their predicted secondary structures (Figure 2) are similar to that of $D$. farinae and $D$. pteronyssinus. As in typical arthropod mtDNA, there is only a small D-loop region between tRNA genes. The D-loop of the $P$. cuniculi mt genome is located between the tRNA-Phe and tRNASer $^{\text {AGN }}$ (Table 3). The size of D-loop is $382 \mathrm{bp}$, and the $\mathrm{A}+\mathrm{T}$ content is $89.8 \%$. This region contains several dinucleotide $[\mathrm{AT}]_{5-40}$ repeats, which are conserved sequences in mt genomes of $D$. farinae and $D$. pteronyssinus $[17,23]$. In addition, this region holds a short palindromic sequence, TACAT, which is usually a conserved motif that is often found in $\mathrm{mt}$ genomes of insects, including $D$. farinae and D. pteronyssinus $[17,23]$.

\section{Phylogenetic analyses}

Of the 51 Acari species included in the phylogenetic analyses in this study, 35 species belonged to the superorder Parasitiformes while 16 belonged to the superorder Acariformes. Both superorders Parasitiformes and Acariformes were monophyletic in all of the trees inferred by the BI, ML and MP methods. The monophyly of the superorder
Parasitiformes was strongly supported with a Bpp of 1 in $\mathrm{BI}$ analyses (Figure 3), a Bf of $81 \%$ in $\mathrm{ML}$ analyses (Figure 4), and a $\mathrm{Bf}$ of $82 \%$ in MP analyses (Figure 5). The monophyly of the superorder Acariformes was strongly supported in $\mathrm{BI}$ and $\mathrm{ML}$ analyses $(\mathrm{Bpp}=1$, Figure 3 ; $\mathrm{Bf}=$ $100 \%$, Figure 4), and was moderately supported in MP analysis $(\mathrm{Bf}=97 \%$, Figure 5$)$. The 35 species of ticks in the superorder Parasitiformes included in this study were from two orders: Ixodida (32 species) and Mesostigmata (3 species). The monophyly of the order Ixodida was strongly supported in BI and ML analyses $(\mathrm{Bpp}=1$, Figure 3; $\mathrm{Bf}=95 \%$, Figure 4$)$, but was weakly supported in MP analysis ( $\mathrm{Bf}=46 \%$, Figure 5$)$. The Mesostigmata were monophyletic with strong support in BI analysis $(\mathrm{Bpp}=1$, Figure 3), and was moderately supported in ML analysis $(\mathrm{Bf}=81 \%$, Figure 4$)$, and weakly supported in MP analysis $(\mathrm{Bf}=56 \%$, Figure 5$)$. Of the 16 species of mites in the superorder Acariformes, 4 species were from the order Astigmata, one species from the order Oribatida and 11 species from the order Prostigmata. The Astigmata was monophyletic with strong support in all of the three phylogenetic analyses $(\mathrm{Bpp}=1$, Figure 3; $\mathrm{Bf}=100 \%$, Figure 4; $\mathrm{Bf}=100 \%$, Figure 5). However, the Prostigmata was not monophyletic in all of the three phylogenetic analyses in the present study (Figures 3, 4 and 5). The

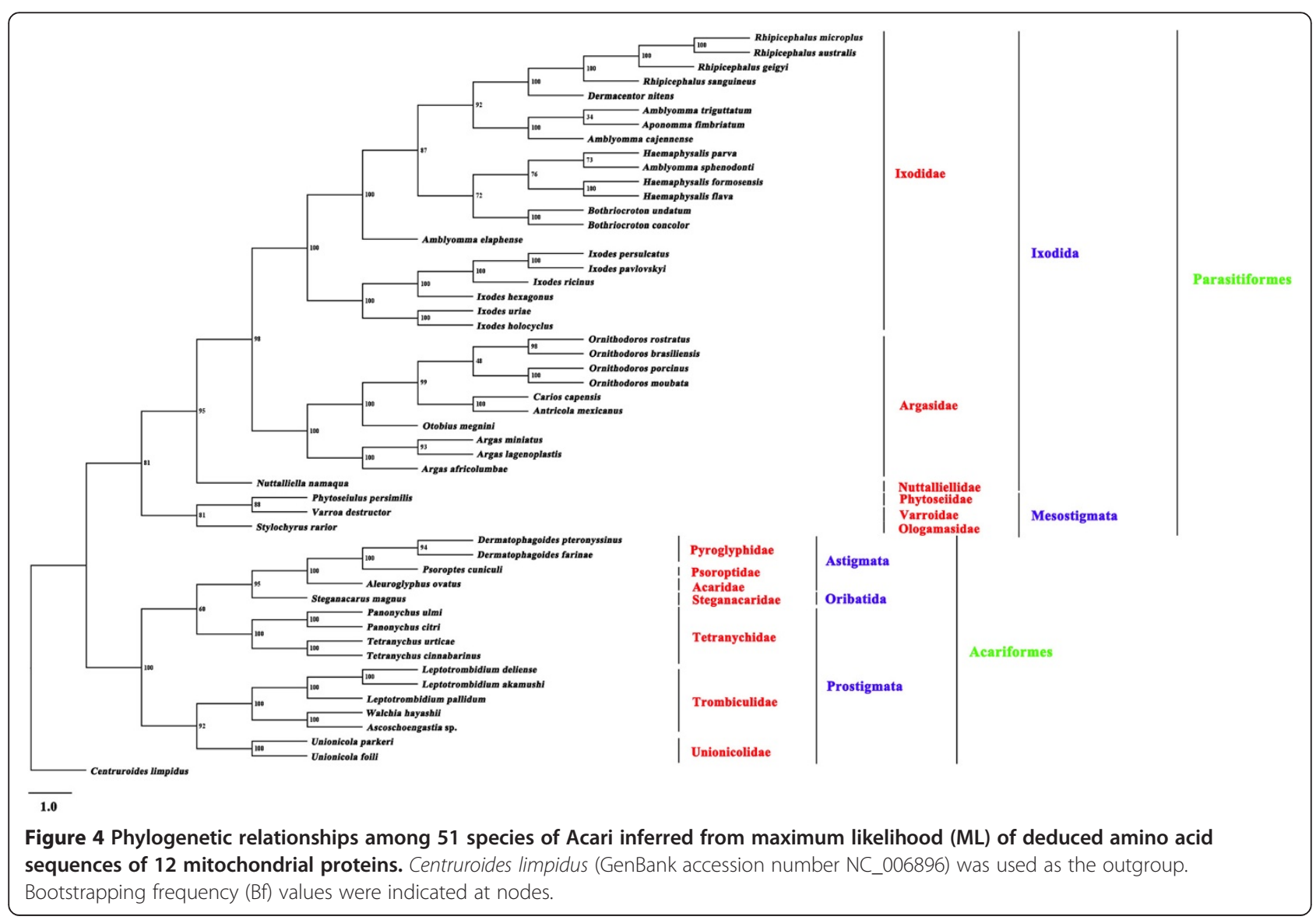




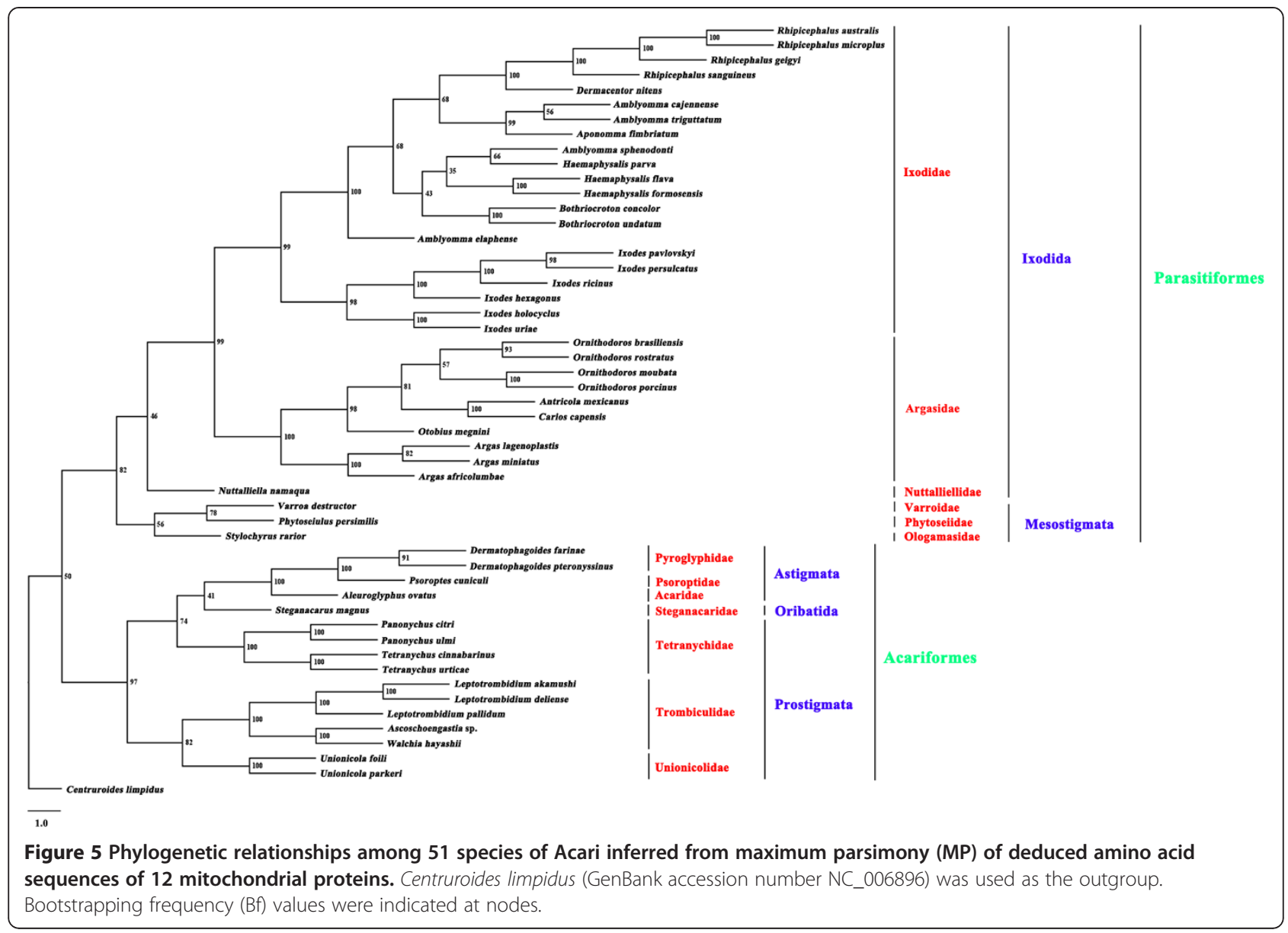

Prostigmata was paraphyletic with respect to the family Tetranychidae. Four species from the Tetranychidae of the Prostigmata were more closely related to families Steganacaridae (order Oribatida), Acaridae, Psoroptidae and Pyroglyphidae (order Astigmata) than they were to the other two families (Trombiculidae and Unionicolidae) of the order Prostigmata. The close relationship between these species of the Tetranychidae and Steganacaridae was strongly supported in $\mathrm{BI}$ analysis $(\mathrm{Bpp}=$ 1 , Figure 3 ), and was moderately supported in $\mathrm{ML}$ and $\mathrm{MP}$ analyses $(\mathrm{Bf}=60 \%$, Figure $4 ; \mathrm{Bf}=74 \%$, Figure 5$)$. In addition to the Prostigmata, four families of the orders Astigmata and Oribatida were also represented in our analyses: Pyroglyphidae (2 species), Psoroptidae (1 species), Acaridae (1 species) and Steganacaridae (1 species). The Pyroglyphidae was monophyletic with strong support in all of the three phylogenetic analyses in this study $(\mathrm{Bpp}=1$, Figure $3 ; \mathrm{Bf}=94 \%$, Figure $4 ; \mathrm{Bf}=91 \%$, Figure 5 ). Of the 32 species of ticks in the Ixodida, 21 species were from the family Ixodidae (hard ticks), 10 species from the family Argasidae (soft ticks) and one species from the family Nuttalliellidae. The two families Ixodidae and Argasidae were monophyletic with strong support in BI analysis $(\mathrm{Bpp}=1$,
Figure 3$), \mathrm{ML}$ analysis $(\mathrm{Bf}=100 \%$, Figure 4$)$ and $\mathrm{MP}$ analysis ( $\mathrm{Bf}>99 \%$, Figure 5). The four species of the genus Amblyomma within Ixodidae were included in this study. These results indicated that Amblyomma is paraphyletic with strong support in BI analysis (Figure 3), and is moderately supported in ML and MP analyses (Figures 3 and 4).

\section{Discussion}

For two decades, there has been considerable debate surrounding the systematics of Acari. The subclass Acari were initially recognized as three lineages (Acariformes, Parasitiformes and Opiloacariformes), however, some authors later demonstrated that there are two distinct groups: Acariformes and Parasitiformes [34-37], and Opiloacariformes as an independent lineage were rejected as they represent basal Parasitiformes [14]. Unfortunately, as there are no complete mt genomes of Opilioacariformes available, we were not able to verify this hypothesis. Dabert et al. [38] confirmed the monophyly of Acariformes based on the nuclear small subunit rRNA gene (18S rDNA) and the mt cytochrome c oxidase subunit I (COX1). Dermauw et al. [17] tested the hypothesis of the Acari phylogeny with the $\mathrm{mt}$ genome sequences of 
21 species, and showed strong support of the monophylies of the superorders Parasitiformes and Acariformes, represented by 12 species from the Parasitiformes and 9 species from the Acariformes. This study, however, could not reject the monophyly of the order Prostigmata. In the present study, our analyses support the division of the subclass Acari into two superorders Parasitiformes and Acariformes [14], however, the order Prostigmata was paraphyletic in all of the three phylogenetic analyses in this study. Although morphological characteristics remain an important source of phylogenetic signal in organismal evolution, morphological characters and molecular characters vary among orders and major groups of Acari. Prostigmata phylogeny based on morphological characteristics shows that the order Prostigmata has an apparent monophyly and is sister to orders Endeostigmata + Oribatids [39]. However, molecular data provide some support that the order Prostigmata was paraphyletic in the current study. However, we should be cautious that the phylogenetic signal may be influenced by long-branch attraction artifacts, this phenomenon may challenge the analyses of phylogeny of Acariform mites [38].

The superorder Parasitiformes is composed of four orders: Opilioacarida, Holothyrida, Ixodida, and Mesostigmata, and the superorder Acariformes is divided into two suborders: Sarcoptiformes (consisting of Endeostigmata, Oribatida and Astigmata) and Trombidiformes (Prostigmata or Actinedida) [40]. Domes et al. (2007) showed that the order Astigmata is located on the outside of the order Oribatida, usually as sister-group of the order Endeostigmata [41]. However, Dabert et al. [38] speculated that order Oribatida is paraphyletic with respect to order Astigmata. In the present study, although our results have shown that the orders Oribatida and Astigmata are sister groups, only one species within the order Oribatida was included in the present study.

In the present study, our results provide strong support that families Ixodidae and Argasidae are sister taxa, consistent with those of previous studies $[4,42]$. Here, we will not discuss the phylogenetic relationships of other families within the superorder Parasitiformes (eg., Nuttalliellidae, Phytoseiidae, Varroidae and Ologamasidae) due to limited data. Phylogenetic analyses based on $\mathrm{mt}$ genome sequences in the present study strongly support a close relationship between families Tetranychidae and Steganacaridae + Acaridae + Psoroptidae + Pyroglyphidae to the exclusion of families Trombiculidae and Unionicolidae. Apparently, additional markers for phylogenetic inference are required to resolve the controversy on the order Prostigmata between phylogeny based on mt genome sequences and nuclear SSU rRNA sequences [15]. The monophyly of the order Oribatida will also need further testing with expanded taxon sampling as only one species, Steganacarus magnus, from the Steganacaridae was included in the present study. No species from the superorder Opilioacariformes and order Holothyrida was included in our analyses. Therefore, expanding taxon sampling from these lineages of Acari is clearly the next step for phylogenetic studies of Acari using mtDNA. In addition, phylogenetic analysis in the present study was based only on mtDNA sequences, so we believe it is still necessary to employ nuclear genomic sequences to provide additional evidence for phylogenetic analyses and genome evolution of the Acari.

\section{Conclusion}

The present study determined the mt genome of the mite species $P$. cuniculi of animal health significance, and represents the first mt genome study of any member of the family Psoroptidae. Phylogenetic analyses of the mt genome sequences of the $P$. cuniculi species, in combination with 50 other Acari species, support the monophylies of the superorders Parasitiformes and Acariformes; the orders Ixodida, Mesostigmata and Astigmata, however, reject the monophyly of the order Prostigmata. Our results indicate the need to test hypotheses of Acari phylogeny with more taxa and different molecular markers.

\section{Competing interests}

The authors declare that they have no competing interests.

\section{Authors' contributions}

XBG, GHL, HQS, TYL and GYY performed the experiments, analyzed the data and drafted parts of the manuscript. XQZ conceived and funded the study, revised and edited the manuscript. All authors read and approved the final manuscript.

\section{Acknowledgements}

This work was supported by the International Science \& Technology Cooperation Program of China (Grant No. 2013DFA31840), the Science Fund for Creative Research Groups of Gansu Province (Grant No. 1210RJIA006) and Sichuan Provincial Department of Education (Grant No. 14ZB0003).

\section{Author details}

${ }^{1}$ State Key Laboratory of Veterinary Etiological Biology, Key Laboratory of Veterinary Parasitology of Gansu Province, Lanzhou Veterinary Research Institute, Chinese Academy of Agricultural Sciences, Lanzhou, Gansu Province 730046, P R China. ²Department of Parasitology, College of Veterinary Medicine, Sichuan Agricultural University, Ya'an, Sichuan Province 625014, P R China.

Received: 15 May 2014 Accepted: 14 July 2014

Published: 22 July 2014

\section{References}

1. Wolstenholme DR: Animal mitochondrial DNA, structure and evolution. Int Rev Cytol 1992, 141:173-216.

2. Boore JL: Animal mitochondrial genomes. Nucleic Acids Res 1999, 27:1767-1780.

3. Tao M, You CP, Zhao RR, Liu SJ, Zhang ZH, Zhang C, Liu Y: Animal mitochondria: evolution, function, and disease. Curr Mol Med 2014, 14:115-124.

4. Burger TD, Shao R, Beati L, Miller H, Barker SC: Phylogenetic analysis of ticks (Acari: Ixodida) using mitochondrial genomes and nuclear rRNA genes indicates that the genus Amblyomma is polyphyletic. Mol Phylogenet Evol 2012, 64:45-55. 
5. Jabbar A, Mohandas N, Jex AR, Gasser RB: The mitochondrial genome of Protostrongylus rufescens-implications for population and systematic studies. Parasit Vectors 2013, 6:263-271.

6. Cameron SL: Insect mitochondrial genomics: implications for evolution for evolution and phylogeny. Annu Rev Entomol 2014, 59:95-117.

7. Liu GH, Gasser RB, Su A, Nejsum P, Peng L, Lin RQ, Li MW, Xu MJ, Zhu XQ: Clear genetic distinctiveness between human- and pig-derived Trichuris based on analyses of mitochondrial datasets. PLOS Negl Trop Dis 2012, 6:e1539.

8. Liu GH, Chen F, Chen YZ, Song HQ, Lin RQ, Zhou DH, Zhu XQ: Complete mitochondrial genome sequence data provides genetic evidence that the brown dog tick Rhipicephalus sanguineus (Acari: Ixodidae) represents a species complex. Int J Biol Sci 2013, 9:361-369.

9. Liu GH, Shao R, Li JY, Zhou DH, Li H, Zhu XQ: The complete mitochondrial genomes of three parasitic nematodes of birds: a unique gene order and insights into nematode phylogeny. BMC Genomics 2013, 14:414-426.

10. Liu GH, Gasser RB, Young ND, Song HQ, Ai L, Zhu XQ: Complete mitochondrial genomes of the 'intermediate form' of Fasciola and Fasciola gigantica, and their comparison with $F$. hepatica. Parasit Vectors 2014, 7:150-159.

11. Walter DE, Proctor HC: Systematic and Morphological Survey. In Mites: Ecology, Evolution \& Behaviour. Edited by Walter DE, Proctor HC. New York: Springer; 2013:39-68.

12. McCoy KD, Léger E, Dietrich M: Host specialization in ticks and transmission of tick-borne diseases: a review. Front Cell Infect Microbiol 2013, 3:57-68

13. Javed S, Khan F, Ramirez-Fort M, Tyring SK: Bites and mites: prevention and protection of vector-borne disease. Curr Opin Pediatr 2013, 25:488-491.

14. Dunlop JA, Alberti G: The affinities of mites and ticks: a review. J Zool Syst Evol Res 2008, 46:1-18.

15. Murrell A, Dobson SJ, Walter DE, Campbell NJH, Shao R, Barker SC: Relationships among the three major lineages of the Acari (Arthropoda: Arachnida) inferred from small subunit rRNA: paraphyly of the Parasitiformes with respect to the Opilioacariformes and relative rates of nucleotide substitution. Invert Syst 2005, 19:383-389.

16. Klompen H, Lekveishvili M, Black WC: Phylogeny of parasitiform mites (Acari) based on rRNA. Mol Phylogenet Evol 2007, 43:936-951.

17. Dermauw W, Van Leeuwen T, Vanholme B, Tirry L: The complete mitochondrial genome of the house dust mite Dermatophagoides pteronyssinus (Trouessart): a novel gene arrangement among arthropods. BMC Genomics 2009, 10:107-127.

18. Burger TD, Shao R, Labruna MB, Barker SC: Molecular phylogeny of soft ticks (Ixodida: Argasidae) inferred from mitochondrial genome and nuclear rRNA sequences. Ticks Tick Borne Dis 2014, 5:195-207.

19. Cutler SL: Ectopic Psoroptes cuniculi infestation in a pet rabbit. J Small Anim Pract 1998, 39:86-87.

20. Zheng WP, Zhang $\mathrm{RH}$, Wu XH, Ren Y, Nong X, Gu XB, Wang SH, Peng XR, Yang GY: Evaluating troponin $C$ from Psoroptes cuniculi as a diagnostic antigen for a dot-ELISA assay to diagnose mite infestations in rabbits. Parasite Immunol 2014, 36:53-59.

21. Sweatman GK: On the life history and validity of the species in Psoroptes, a genus of mange mites. Can J Zool 1958, 36:905-929.

22. Folmer $\mathrm{O}$, Black M, Hoeh W, Lutz R, Vrijenhoek R: DNA primers for amplification of mitochondrial cytochrome c oxidase subunit I from diverse metazoan invertebrates. Mol Mar Biol Biotechnol 1994, 3:294-299.

23. Klimov PB, OConnor BM: Improved tRNA prediction in the American house dust mite reveals widespread occurrence of extremely short minimal tRNAs in acariform mites. BMC Genomics 2009, 10:598-616.

24. Katoh K, Standley DM: MAFFT multiple sequence alignment software version 7: improvements in performance and usability. Mol Biol Evol 2013, 30:772-780

25. Tamura K, Peterson D, Peterson N, Stecher G, Nei M, Kumar S: MEGA 5: molecular evolutionary genetics analysis using maximum likelihood, evolutionary distance, and maximum parsimony methods. Mol Biol Evol 2011, 28:2731-2739.

26. Lowe TM, Eddy SR: tRNAscan-SE: a program for improved detection of transfer RNA genes in genomic sequence. Nucleic Acids Res 1997, 25:955-964.

27. Laslett D, Canback B: Arwen: a program to detect tRNA genes in metazoan mitochondrial nucleotide sequences. Bioinformatics 2008, 24:172-175.
28. Dávila S, Piñero D, Bustos P, Cevallos MA, Dávila G: The mitochondrial genome sequence of the scorpion Centruroides limpidus (Karsch 1879) (Chelicerata; Arachnida). Gene 2005, 360:92-102.

29. Talavera G, Castresana J: Improvement of phylogenies after removing divergent and ambiguously aligned blocks from protein sequence alignments. Syst Biol 2007, 56:564-577.

30. Abascal F, Zardoya R, Posada D: ProtTest: selection of best-fit models of protein evolution. Bioinformatics 2005, 21:2104-2105.

31. Ronquist F, Huelsenbeck JP: MrBayes 3: Bayesian phylogenetic inference under mixed models. Bioinformatics 2003, 19:1572-1574.

32. Guindon S, Gascuel O: A simple, fast, and accurate algorithm to estimate large phylogenies by maximum likelihood. Syst Biol 2003, 52:696-704.

33. Swofford DL: PAUP*: Phylogenetic Analysis Using Parsimony, Version 4.0610 Sunderland: Sinauer Associates; 2002.

34. Alberti G: Zur Feinstruktur der Spermien und Spermiocytogenese der Milben (Acari) I. Anactinotrichida. Zool Jb Anat 1980, 104:77-138.

35. Alberti G: Zur Feinstruktur der Spermien und Spermiocytogenese der Milben (Acari) II. Actinotrichida. Zool Jb Anat 1980, 104:144-203.

36. Lindquist EE: Current Theories on the Evolution of Major Groups of Acari and on their Relationships with other Groups of Arachnida with Consequent Implications for their Classification. In Acarology. Volume 1. Edited by Griffiths DA, Bowman CE. Chichester: Ellis Horwood Ltd; 1984:28-62.

37. Bernini F: Current ideas on the phylogeny and the adaptive radiations of Acarida. Boll Zool 1986, 53:279-313.

38. Dabert M, Witalinskib W, Kazmierskic A, Olszanowskid Z, Dabertc J: Molecular phylogeny of acariform mites (Acari, Arachnida): Strong conflict between phylogenetic signal and long-branch attraction artifacts. Mol Phylogenet Evol 2010, 56:222-241.

39. Jeffrey WS: A phylogenetic analysis of the arachnid orders based on morphological characters. Zool J Linn Soc 2007, 150:221-265.

40. Lindquist EE, Krantz GW, Walter DE: Classification. In A Manual of Acarology. 3rd edition. Edited by Krantz GE, Walter DE. Lubbock: Texas Tech University Press; 2009:97-103.

41. Domes K, Althammer M, Norton RA, Scheu S, Maraun M: The phylogenetic relationship between Astigmata and Oribatida (Acari) as indicated by molecular markers. Exp Appl Acarol 2007, 42:159-171.

42. Mans BJ, Klerk D, Pienaar R, de Castro MH, Latif AA: The Mitochondrial genomes of Nuttalliella namaqua (Ixodoidea: Nuttalliellidae) and Argas africolumbae (Ixodoidae: Argasidae): estimation of divergence dates for the major tick lineages and reconstruction of ancestral blood-feeding characters. PLoS One 2012, 7:e49461.

\section{doi:10.1186/1756-3305-7-340}

Cite this article as: Gu et al:: The complete mitochondrial genome of the scab mite Psoroptes cuniculi (Arthropoda: Arachnida) provides insights into Acari phylogeny. Parasites \& Vectors 2014 7:340.

\section{Submit your next manuscript to BioMed Central and take full advantage of:}

- Convenient online submission

- Thorough peer review

- No space constraints or color figure charges

- Immediate publication on acceptance

- Inclusion in PubMed, CAS, Scopus and Google Scholar

- Research which is freely available for redistribution 